\section{Pulmonary Sequestration With Abdominal Aorta Feeding Vessel in an Infant}

\author{
Haisong Bu, MD; Tianli Zhao, MD
}

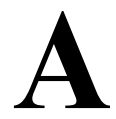
9-month-old girl from a remote village presenting with recurrent cough and repeated pneumonia was referred to the present institution for ventricular septal defect (VSD) closure and atrial septal defect (ASD) closure. Physical examination indicated reduced arterial oxygen saturation ( $86 \%$ at room temperature, not corrected by oxygen support) and mild cyanosis. A grade $3 / 6$ systolic murmur was audible at the third left intercostal space. Transthoracic echocardiography at the local county hospital indicated VSD, ASD and pulmonary hypertension. On 3-D computed tomography angiography, a dilated and abnormal right superior pulmonary vein (RSPV; Figure A-C, white arrow), ASD, VSD (Figure A,B) and one aberrant artery arising from the abdominal aorta and supplying sequestration on the right side, were seen (Figure C; red arrow). Digital subtraction angiography showed a systemic artery arising from the abdominal aorta supplying the right lower lobe sequestration (Figure D, red arrow) and the absence of the right lower pulmonary artery (Figure E). Following communication with the patient's parents, embolization (Figure F, red arrow) and thoracotomy were requested. Subsequently, the right lower pulmonary lobe and the heart defects were surgically removed and repaired, respectively.

This report highlights the essence of increasing the preoperative diagnosis rate in Chinese rural areas. Clinical presentation of pulmonary sequestration varies, and preoperative diagnosis is often incorrect. A clear early onset age, recurrent pneumonia, mass or cyst lesion located in the lower lobe, and aberrant arterial supply are indicators of the diagnosis.

\section{Acknowledgment}

We thank the patient for participating in this study.

\section{Disclosures}

The authors declare no conflict of interest.

Received August 2, 2018; revised manuscript received October 24, 2018; accepted November 17, 2018; J-STAGE Advance Publication released online December 20, 2018 Time for primary review: 82 days

Department of Cardiovascular Surgery, Second Xiangya Hospital, Central South University, Changsha, China

Mailing address: Tianli Zhao, MD, Department of Cardiovascular Surgery, The Second Xiangya Hospital, Central South University, 139 Renmin Central Road, Changsha, Hunan 410011, China. E-mail: zhaotianli@csu.edu.cn

ISSN-1346-9843 All rights are reserved to the Japanese Circulation Society. For permissions, please e-mail: cj@j-circ.or.jp
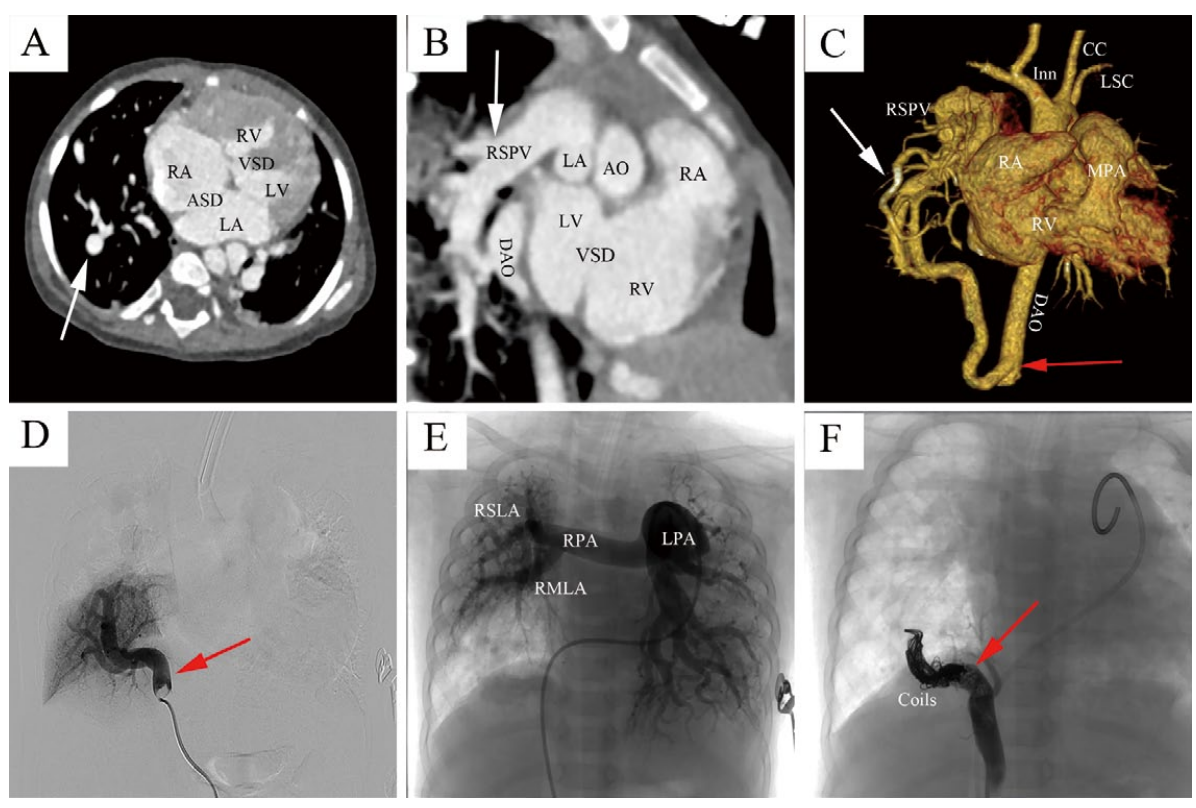

Figure. Imaging of pulmonary sequestration. (A) Computed tomography (CT) showing a ventricular septal defect (VSD), atrial septal defect (ASD) and dilated right superior pulmonary vein (RSPV, arrow). (B) Dilated and abnormal RSPV (arrow) draining the right lobe. (C) Angio-CT reconstruction confirming the presence of a single arterial feeding vessel of the pulmonary sequestration (arrow). (D) Angiography demonstrating a systemic arterial feeder (arrow) to the pulmonary sequestration in the right lower lobe. (E) Pulmonary artery angiography showing absence of the right lower lobe artery. (F) The aberrant artery was embolized with coils (arrow). AO, aorta; CC, left common carotid artery; DAO, descending aorta; Inn, innominate artery; LA, left atrium; LPA, left pulmonary artery; LSC, left subclavian artery; LV, left ventricle; MPA, main pulmonary artery; RA, right atrium; RMLA, right middle lobe artery; RPA, right pulmonary artery; RSLA, right superior lobe artery; RV, right ventricle. 